(2) Open Access Full Text Article

REVIEW

\title{
Impact of obstructive sleep apnea on blood pressure in patients with hypertension
}

\author{
This article was published in the following Dove Press journal: \\ ChronoPhysiology and Therapy \\ 30 November 2011 \\ Number of times this article has been viewed
}

\author{
Barry Fields' \\ Indira Gurubhagavatula ${ }^{1-3}$ \\ 'Division of Sleep Medicine, Perelman \\ School of Medicine, University of \\ Pennsylvania, ${ }^{2}$ Center for Sleep and \\ Circadian Neurobiology, Perelman \\ School of Medicine, University of \\ Pennsylvania, ${ }^{3}$ Philadelphia VA Medical \\ Center, Philadelphia, Pennsylvania, \\ USA
}

\begin{abstract}
Hypertension is the most significant risk factor for death worldwide. Approximately $30 \%-40 \%$ of affected individuals have coexisting obstructive sleep apnea (OSA), a disorder resulting from the upper airway's inability to remain patent during sleep. A causal relationship between OSA and hypertension has been demonstrated. Blunting or elimination of normal blood pressure (BP) dipping during sleep is commonly seen in OSA patients, with corresponding increases in daytime BP. This dipping is clinically salient, because it is associated with the endorgan damage seen with chronic hypertension, such as cardiovascular, renal, and cerebrovascular disease. African-Americans are at greatest risk for non-dipping and end-organ damage. Rapidly fluctuating changes in sympathetic tone, intrathoracic pressure, oxyhemoglobin saturation, and carbon dioxide levels are all thought to play a role in acute and chronic BP elevation. Individuals with preexisting hypertension are most susceptible to OSA's BP-raising effects. First-line therapy for OSA includes continuous positive airway pressure (CPAP) delivered via a mask interface. Patients who show the greatest BP declines while using CPAP are more likely to be those who have at least moderate OSA, adhere to therapy, have preexisting hypertension, and whose blood vessels retain reversibility in disease-related remodeling. Given the heavy burden OSA-related hypertension places on the healthcare system, prevention, early detection, and prompt intervention should be the goals for all affected individuals.
\end{abstract}

Keywords: obstructive sleep apnea (OSA), hypertension, nocturnal dipping, continuous positive airway pressure (CPAP)

\section{The coexistence of sleep apnea and hypertension Hypertension: a worldwide epidemic}

In 2004, the Joint National Committee on Prevention, Evaluation, and Treatment of High Blood Pressure produced its seventh Report. "JNC-7" reviewed the extraordinary impact hypertension has on the world population, citing World Health Organization data that attributed $\sim 7.1$ million deaths/year to hypertension. Specifically, the World Health Organization asserted that $62 \%$ of cardiovascular disease, including stroke, and $49 \%$ of ischemic heart disease result from hypertension, estimates that make poorly controlled blood pressure (BP) the most significant risk factor for death worldwide. ${ }^{1}$ Since up to one billion people have hypertension (defined as a BP $>140 / 90$ in most individuals, or a BP $>130 / 80$ in diabetics and patients with kidney disease), the public health burden is enormous. ${ }^{2}$ Control of blood pressure and prevention of incident cases necessitates risk factor reduction in conjunction with therapeutic interventions.

Much of the analysis detailed by JNC-7 centers on hypertension's participation in the metabolic syndrome, a multisystem disorder including obesity, impaired fasting
Correspondence: Barry Fields Division of Sleep Medicine, 3624 Market Street, Suite 205, Philadelphia, PA 19104, USA

Tel +I 2I5-6I5-4875

Fax +| 2|5-6|5-4874

Email barry.fields@uphs.upenn.edu 
glucose, hypertriglyceridemia, and reduced high-density lipoprotein (HDL, so-called "good") cholesterol plaguing nearly one quarter of Americans. ${ }^{3}$ Though not included in this syndrome, one of many comorbidities of metabolic syndrome is obstructive sleep apnea (OSA). JNC-7 highlights this disorder as another risk factor for developing hypertension, independent of established metabolic syndrome contributors. In fact, of the $2 \%-4 \%$ of the world estimated to have OSA, about half suffer from hypertension. ${ }^{46}$

\section{Characterizing obstructive sleep apnea}

OSA results from the upper airway's inability to remain patent during sleep regardless of the diaphragm's efforts to breathe; obesity (body mass index $>30 \mathrm{~kg} / \mathrm{m}^{2}$ ) is its greatest risk factor. Oxyhemoglobin saturation levels promptly fall during these events, stimulating a brief arousal from sleep. This breathing interruption (termed an "apnea" if breathing fails to resume after 10 seconds) typically recurs throughout the night. BP initially falls at the start of each episode but then climbs gradually until respiration returns following a brief arousal from sleep. Systolic BP can rise by 15 to $80 \mathrm{mmHg}$ during arousals before swinging back to baseline levels, placing undulating stress on the heart, lungs, and other end-organs. ${ }^{7}$ These apneas can result in the OSA syndrome, a constellation of symptoms including loud snoring, choking or gasping during sleep, and excessive daytime sleepiness. Other symptoms include nocturia, depression, decreased libido, and decline in memory and concentration.

While "apneas" refer to total airflow obstruction, hypopneas are defined by a decrease in airflow for at least 10 seconds that is terminated by either arousal from sleep or at least a $3 \%$ to $4 \%$ oxyhemoglobin desaturation. ${ }^{8}$ Hypopneas have been shown to produce the same cardiovascular consequences as apneas. ${ }^{9}$ Grading the severity of OSA requires counting an individual's apneas and hypopneas and averaging this number over their hours of sleep, producing an "Apnea-Hypopnea Index" or AHI. An AHI $\geq 5$ events/hour is the designated threshold for at least mild OSA, while an AHI $\geq 15$ events/hour delineates at least moderate disease. An AHI $\geq 30$ events/hour defines severe OSA. As we will see later, it is the group with moderate to severe disease that carries the greatest risk of having hypertension, and also has the greatest chance to experience $\mathrm{BP}$ reduction if the OSA is treated.

\section{Diurnal blood pressure variations Nocturnal dipping}

During sleep, blood pressure decreases from waking values, a process known as "nocturnal dipping." While there is considerable variability in the degree of this drop among individuals, both systolic and diastolic pressures typically decline $10 \%$ to $15 \% .^{10}$ This dip appears to be a function of sleep-wake state rather than circadian (or "nocturnal") rhythm; sleep deprived, healthy subjects retain nocturnal drops in heart rate but maintain daytime BP values at night unless they are allowed to sleep. ${ }^{11}$ Therefore, while "nocturnal dipping" may be an imprecise term when applied to BP, its use is ubiquitous.

The human autonomic nervous system plays a key role in BP decline during sleep. As individuals fall asleep, they typically transition from wakefulness to non-rapid eye movement (NREM) sleep. Constituting the majority of sleep time, NREM sleep is a period of remarkable cardiovascular stability. Alerting sympathetic neural responses wane, and the parasympathetic system exerts its primary effects: cardiac output and BP fall due to decreased heart rate and systemic vascular resistance. Deep sleep sympathetic neural activity is reduced to just half of normal wakefulness levels. ${ }^{12}$ The hypothalamic pituitary axis also slows, with serum catecholamine levels typically at their lowest during deep sleep. Rapid eye movement (REM) sleep punctuates parasympatheticallydominated NREM sleep periods increasingly throughout the night, producing sympathetic surges that transiently elevate $\mathrm{BP}$ and heart rate. Therefore, any condition that blunts the body's normal BP dips during NREM or exacerbates expected BP elevation during REM exposes an individual to higher BPs throughout the night.

One such condition is comorbid OSA in individuals with hypertension. An observational study utilizing 24 hour ambulatory BP monitoring indicated that while individuals with coexisting OSA and hypertension were "non-dippers" in terms of nocturnal BP, normotensive OSA patients maintained their nocturnal dipping. ${ }^{13}$ Additional work has shown that African-Americans are more likely than Caucasians to be non-dippers independent of OSA status, but both races tend to be non-dippers when OSA and hypertension coexist. Only in African-Americans does OSA severity directly correlate with non-dipper status. ${ }^{14}$

The presence of this nocturnal dipping is salient, as several studies showed that its absence increases risk of stroke, heart failure, and renal disease. ${ }^{15-18}$ A Japanese study quantified this relationship further: every $5 \%$ loss in "nocturnal dipping" of BP was associated independently with a $20 \%$ increase in cardiovascular mortality. ${ }^{19}$

That even a small blunting of nocturnal dipping has significant health consequences seems unsurprising when considered in the context of studies measuring overall BP. 
Even small reductions in average BP can greatly reduce cardiovascular morbidity and mortality: one 2009 metaanalysis examining 147 randomized trials of anti-hypertensive agents showed a $41 \%$ decline in stroke and a $22 \%$ decline in coronary artery disease events from just a $10 \mathrm{mmHg}$ reduction in systolic BP or a $5 \mathrm{mmHg}$ reduction in diastolic $\mathrm{BP}^{20}$ More modest but still significant declines in stroke (15\%) and coronary heart disease risk (6\%) associated with just a $2 \mathrm{mmHg}$ reduction in diastolic BP have been described. ${ }^{21}$

\section{Challenges to normal nocturnal dipping}

The lowering of BP during the nocturnal dip may have putative benefits, and efforts have been made to quantify the duration of sleep needed to achieve dipping. Multiple surveys within the industrialized world show humans to be sleeping less, and evidence is growing that this phenomenon has deleterious effects on BP. One observational study of 4810 middle-aged individuals showed that diminished sleep time (less than 5 hours) was associated with $60 \%$ higher rates of hypertension in the 8-10 years of follow-up, even with tight control of potential confounding variables. ${ }^{22}$ More recent data support these findings and suggest that each 1 hour reduction in sleep duration increases the odds of developing hypertension by $37 \% .{ }^{23}$ Interestingly, this association between the incidence of hypertension and shortened sleep duration has not been found in individuals over age 60 .

In addition to recognizing the impact of sleep duration on nocturnal dipping and daytime BP, one must also consider quality. Several sleep disorders that fragment sleep and degrade its quality have been associated with elevated BP during the day and night such as insomnia, restless legs syndrome, and periodic limb movements. ${ }^{24}$ OSA has been the best studied disorder in its association with hypertension, both at the population and at the biochemical level. Examining the pathophysiology underpinning OSA's link to hypertension helps explain population-based observations of their association.

OSA leads to a self-perpetuating cycle of low oxygenation (hypoxemia), increasing carbon dioxide levels (hypercapnia), and increasingly negative intrathoracic pressure against an occluded airway. These events terminate in arousals, which results in sleep fragmentation and overall poor sleep quality. Recurrent hypoxia has been implicated as the primary stimulus for nocturnal sympathetic nervous system activation, a loss of nocturnal dipping, and ultimately, hypertension. An elegant animal model developed in the 1990s illustrated this point. Brooks et al produced sleep apnea in dogs by closing a previously implanted tracheal valve intermittently during sleep. These dogs experienced acute periods of nocturnal BP elevation, followed by chronic sustained daytime BP elevation. Six months after this experimental protocol ended, the same dogs were awakened the same number of times by a simple tone instead of airway occlusion. While nocturnal BP still rose, there was no sustained increase in BP during the daytime, suggesting that persistent daytime BP elevation due to OSA is a function of hypoxia and/or intrathoracic pressure fluctuation rather than sleep fragmentation per se. ${ }^{25}$

The results of human studies lend further credence to findings in animal models. A small study exposed 21 healthy, awake individuals to a 20 second apnea each minute for 30 minutes. About half the group breathed room air (21\% oxygen) the rest of time, while the other half breathed gas with only $10 \%$ oxygen. The investigators determined that the group with accentuated hypoxemia showed significant increases in sympathetic activation and higher BP; neither outcome measure showed any increases in the group breathing room air. ${ }^{26}$

Older adults suffer moderate to severe OSA rates of at least $20 \%,{ }^{27}$ and are particularly sensitive to BP changes (or lack thereof) associated with blunted nocturnal dipping. Endeshaw et al sought to examine the relationship between OSA and 24 hour BP in a sample of elderly adults aged 65 or over. Of the 69 individuals participating in the study, 20 $(29 \%)$ had moderate to severe OSA. Nocturnal hypertension was more common in this group than in those individuals without OSA or with only mild disease. The investigators also found blunted nocturnal BP dipping in the former group as opposed to the later, even after controlling for posture, daytime activities, and nighttime urination. ${ }^{28}$

\section{The association of hypertension with obstructive sleep apnea Epidemiologic evidence}

More than 2 decades' worth of research shows a significant association between hypertension and obstructive sleep apnea. Data since the 1980s indicates that approximately half of patients with known OSA have coexisting hypertension, and between $30 \%$ and $40 \%$ of patients with hypertension have OSA. ${ }^{29-31}$ The proportion is even greater when considering patients only with resistant hypertension, a recalcitrant form of the disorder that requires at least three medications including a diuretic to control. In this population, up to $85 \%$ of patients have been found to have at least mild OSA. ${ }^{32} \mathrm{~A}$ recent review speculates that hyperaldosteronism-induced pharyngeal edema may be a mediator. ${ }^{33}$ Interestingly, additional 
work suggests that the association between hypertension and OSA is particularly strong in symptomatic patients who report daytime sleepiness, as opposed to patients diagnosed with OSA without any subjective symptoms of the disease. One hypothesis as to why this may occur will be discussed later in the "Management" section. ${ }^{34}$

The first study to explore the association between OSA and hypertension in a general population of men and women previously undiagnosed with either condition took place in 2000. Participants were first screened for symptoms of OSA, with individuals screening positive undergoing overnight polysomnography (PSG; a "sleep study" used as the current gold standard to diagnose OSA). After controlling for confounding factors such as age, body mass index, sex, race, alcohol use, and smoking, OSA was shown to be independently associated with hypertension. This relationship was strongest in younger adult subjects and in individuals with more severe obstructive disease. ${ }^{35}$ The pediatric literature also shows that younger individuals demonstrate this correlation most prominently. New findings indicate that school aged children with OSA of any severity experience 10 to $15 \mathrm{mmHg}$ BP elevations during sleep and wakefulness. ${ }^{36}$ The ramifications of OSA related hypertension in this population, whose rates of obesity are rising, require further investigation.

Refocusing on adults, several studies have gone on to demonstrate a linear, dose-response relationship between OSA and hypertension. An Israeli population-based study of 2677 individuals revealed that not only was OSA associated with hypertension after controlling for age, BMI, and sex, but that a dose-response relationship does exist. Lavie et al found that each increase in AHI of one point - the addition of just one respiratory event per each hour of sleep - increased the risk of having hypertension by $1 \% .{ }^{37}$ Data from the Wisconsin Sleep Cohort, a population-based study of the natural history of sleep disordered breathing in adults, also showed a linear relationship between AHI and BP through 24 hour ambulatory monitoring. Indeed, an individual with an AHI of 15 - straddling the border of mild and moderate OSA - has twice the odds of having hypertension compared with a person with no OSA $(\mathrm{AHI}=0) .{ }^{38,39}$

Despite a number of supporting studies, conflicting data exist. In 2009, the Sleep Heart Health Study published results from its analysis of 2470 patients with OSA, which failed to find any significantly increased risk of incident hypertension at their 5 year follow-up, even in patients with an AHI $>15 .{ }^{40}$ Recently published data from Spain replicated these findings in a cohort of 1180 adults between age 30 and 70 followed for 7.5 years. ${ }^{41}$ Reconciling these results with overwhelming observational data on this association has proved to be challenging. One author from the Wisconsin Sleep Cohort proposes that, among other factors, differences in AHI measurement and scoring methodology may explain some of the discrepancy. Additionally, the disparate outcomes might be partially explained by age of the study population. For example, subjects in the Wisconsin Sleep Cohort average 47 years in age, whereas Sleep Heart Health Study participants average 60 years. There is some evidence that the OSA-hypertension association may be blunted in older populations. ${ }^{42}$

\section{Potential mechanisms of association}

Considering the preponderance of the evidence, most experts agree that OSA is not only associated with hypertension but plays a causal role in its development. Identifying the intermediary steps between hypoxemia and acute BP elevation observed in OSA patients has long been the focus of investigation. While the exact pathways are yet to be confirmed, activation of a number of neural, humoral, metabolic, and inflammatory processes are all thought to play a role.

$\mathrm{BP}$ elevation is related to an elevation in peripheral vascular resistance (PVR). PVR is, in turn, determined by interplay of circulating vasoactive agents (which may either dilate or contract vessel walls) and the compliance of vessel walls themselves. Over time, vascular walls may become thickened, hardened, and less compliant because of remodeling and fibrosis, which increases PVR. These dilating and constricting factors, in the presence or absence of vessel stiffness, determine which way the balance will tip; dilation lowers PVR and BP, while constriction raises PVR and BP.

In the acute setting, recurrent episodes of hypoxemia, hypercapnia, and increasingly negative intrathoracic pressure lead to increased levels of endothelin (a potent vasoconstrictor), an effect seen more in OSA patients than in control subjects. ${ }^{43}$ This hypoxemia also increases circulating reactive oxygen species which can induce vascular inflammatory responses. Although the pathogenesis is uncertain, these species are thought to blunt the small vessel vasodilatory effect of neurotransmitters such as acetylcholine. ${ }^{44}$ Newly published research demonstrates the acute nature of this oxidative stress, showing significant declines in lipid peroxidation after just one night of OSA treatment in patients with severe disease. ${ }^{45}$

There is also evidence that reactive oxygen species interfere with the vasodilating agent nitric oxide, decreasing its bioavailability and leading to poor BP regulation. One study showed that nitric oxide levels were not only inversely related to OSA severity, but that levels rose after patients 
began treatment for their OSA. ${ }^{46}$ This effect has been shown to take place in as little as 2 nights. ${ }^{47}$ Bayram et al have shown that flow-mediated dilatation of the brachial artery, a process shown to be heavily influenced by nitric oxide, actually increases after 6 months of OSA treatment. With better arterial dilatation, reduction in overall BP would be expected..$^{48}$

But how does intermittent, nocturnal hypoxia continue exerting its prohypertensive effect during the daytime, hours after any apneic periods? This question has led to exploration of mechanisms underpinning chronic BP elevation in patients with OSA. Some researchers theorize that there is pathologic activation within the central sympathetic nervous system in structures such as the hypothalamus, as well as abnormal augmentation at peripheral hypoxemiasensing sites such as the carotid sinus. Accentuated levels of circulating neuromodulators such as angiotensin II and endothelin have been implicated in making these central and peripheral chemoreceptors unusually sensitive to oxygen level changes in hypertensive OSA patients. ${ }^{49}$ Carlson et al showed that these findings may be generalized to normotensive OSA patients, and demonstrated a $40 \%$ decrease in vascular conductance (a measure of vessel narrowing) after angiotensin II infusion. ${ }^{44}$ Healthy control subjects showed no conductance change with the same infusion, suggesting increased sensitivity to this vasoconstrictor in the blood vessels of many OSA patients. ${ }^{50}$ This inappropriate increase in sympathetic tone has been implicated in helping maintain daytime BP elevation despite termination of nocturnal hypoxic events.

That sympathetic nervous system activation underlies both acute and chronic elevations in OSA-associated hypertension has been supported further in several continuous positive airway pressure (CPAP; to be discussed more fully later) treatment trials. Mills et al completed a randomized controlled trial evaluating 50 individuals with AHI $>15$ and varied levels of hypertension (off all medication) before and after just 2 weeks of CPAP therapy. An experimental group received therapeutic CPAP pressures, while the control groups received either subtherapeutic CPAP or nocturnal oxygen alone. In addition to decreased BP, subjects in the treatment group manifested greater drops in sympathetic nervous system-stimulated plasma norepinephrine levels, and daytime and nighttime urinary norepinephrine excretion. ${ }^{51}$ Interestingly, withdrawal of CPAP almost immediately resulted in significant increases in both daytime and 24 hour urinary norepinephrine excretion and afternoon systolic BP. ${ }^{52}$ The longstanding effects of inadequately controlled OSA on the sympathetic nervous system has been demonstrated even in individuals with normal BP; Narkiewicz et al showed that 20 normotensive patients with OSA manifested abnormally elevated sympathetic nervous system activity for up to 6 months after commencing CPAP treatment. ${ }^{53}$

Much of this discussion has focused on hypoxemia as the primary culprit, releasing a variety of effects described previously. We should note, however, that other components of acute apnea have also been implicated in its link to hypertension. One randomized trial treated moderately severe OSA patients with therapeutic CPAP, sham CPAP, or supplemental oxygen. Despite normalizing oxygen levels, patients receiving only supplemental oxygen did not experience significant BP reductions. Therefore, when hypoxemia was corrected but intrathoracic pressure changes were not, BP elevations persisted, suggesting that intrathoracic pressure swings may have an etiological role in the development of hypertension. However, CPAP was only modestly successful, suggesting that pressure changes alone are not sufficient to explain the development of hypertension. These findings suggest the presence of additional mechanisms, other than pressure swings and hypoxia-mediated mechanisms, in producing increased blood pressure. ${ }^{54}$ As we mentioned, sympathetic nervous system activation as well as hypercapnia, dramatic swings in intrathoracic pressure, and hypoxemia may all interact to initiate and perpetuate sustained BP elevation.

\section{Management approaches Overview}

If OSA leads to hypertension in a dose-dependent manner in a large number of adults, and if modestly elevated BP increases cardiovascular risk, then treating OSA should be of paramount importance. Nevertheless, showing that adequately treated OSA significantly reduces BP in patients has been surprisingly difficult.

OSA is treated based on disease severity and patient preference. Options for treating mild OSA include weight loss, positional therapy, oral mandibular-advancement appliances, surgery to remove upper airway soft tissue, or CPAP administered during sleep through various types of facial mask interfaces. For individuals suffering from moderate to severe OSA, CPAP is the treatment of choice. This device works as a pneumatic splint to prevent airway collapse during sleep. ${ }^{55}$ Numerous trials have shown CPAP to be effective in this regard, with significant reduction of AHI serving as a routine index of treatment success. The success of CPAP in lowering BP has been studied more rigorously 
than reduction due to other treatment methods, but results are somewhat mixed.

\section{The role of continuous positive airway pressure}

One meta-analysis from 2007 compared 16 randomized trials with a combined patient pool of 818 individuals. In comparing participants who used CPAP for 2 weeks with a control group who did not use it, the study showed a $2.46 \mathrm{mmHg}$ decline in systolic pressure in the intervention group. Diastolic pressure fell by $1.83 \mathrm{mmHg}$. Although the effect was small, declines in BP were statistically significant. ${ }^{56} \mathrm{~A}$ trial published after this meta-analysis continued to show small but significant diastolic BP declines after 1 month of CPAP use in patients with coronary artery disease, a population particularly vulnerable to increased BP. ${ }^{57}$ However, Alajmi et al published meta-analysis findings showing even smaller, statistically insignificant, drops in BP for the intervention group. ${ }^{58}$ Two additional meta-analyses included only studies that had measured 24 hour ambulatory BP, as opposed to office-based measurements as in the prior two analyses. Once again, BP decline in CPAP users was either insignificant or quite modest (1-2 mmHg) over the 24 hours. ${ }^{59,60}$

Perhaps these meta-analyses raise more questions than they answer. For instance, does first line therapy (CPAP) for moderate to severe OSA really have any significant impact on hypertension and the dangerous cardiovascular consequences resulting from it? Considering subgroup analysis and general methodologies employed may help explain some of the variability in results. First, we note that these trials of CPAP were of limited duration (2 weeks to 6 months). Longitudinal follow-up for much longer periods of time in larger cohorts is essential, particularly in community-based populations. Such trials would help demonstrate whether any initial gains in BP reduction would be maintained over time, and whether such reduction translates to reduced cardiovascular risk.

Other methodological questions concern small sample sizes (only four of the most recent 23 studies included over 100 patients), a relatively high male to female ratio, and most studies' involving just one institution (typically a tertiary referral center) without drawing samples broadly from the general community. Additionally, method of BP measurement varied significantly, yet this value was the primary outcome measure in most studies. Some trials used 24 hour ambulatory BP monitoring in their analysis, while others used office BP measurement. In addition to 24 hour monitoring's superiority to office measurement in terms of daytime accuracy, 24 hour monitoring enables investigators to take nocturnal dipping (or lack thereof) into account.

One recent randomized control trial sought to address some of these limitations. Durán-Cantolla et al studied 340 patients with recently diagnosed, untreated hypertension and untreated moderate to severe OSA at eleven centers throughout Spain. Participants were randomly assigned either to treatment-level CPAP or sham CPAP (with very minimal air pressure) for 3 months. They averaged age 52.4 years with an AHI of 43.5 (with any AHI $>30$ indicating severe disease). Participants underwent 24 hour blood pressure monitoring to determine changes in daytime and nighttime $\mathrm{BP}$ as the study progressed. After 3 months, individuals in the CPAP treatment group achieved a $1.5 \mathrm{mmHg}$ decline in their mean 24 hour BP and a $2.1 \mathrm{mmHg}$ decline specifically in nighttime BP, defined as those measurements taken between $10 \mathrm{pm}$ and $6 \mathrm{am} .{ }^{61}$ Despite these relatively modest declines, as little a BP decrease as $2 \mathrm{mmHg}$ may reduce cardiovascular risk. ${ }^{62}$

The two meta-analyses from 2007 may provide at least some hints as to which populations benefit most from CPAP therapy. They showed greatest BP declines in patients with severe OSA (AHI > 30) and in individuals with baseline hypertension, suggesting CPAP therapy may have its greatest impact on these groups. Additionally, subgroup analysis shows the longer subjects used their CPAP machine each night, the greater effect it had on overall BP reduction. In other words, adherence to CPAP therapy is related to the degree of BP-lowering. ${ }^{58,60}$

This point may seem obvious, but CPAP use is often suboptimal even in research trials. In fact, one meta-analysis showed participants used their CPAP only 4-5 hours per night, leaving them untreated for the remainder of their sleep duration. In the general population, CPAP non-adherence (defined as CPAP use less than 4 hours) ranges between $46 \%$ and $83 \%$ of all users. ${ }^{63}$ Sleepiness may predict those who are more likely to comply; those who experience improvement in baseline hypersomnolence may be more likely to use CPAP. Some recent evidence supports that symptomatic improvement correlates with degree of BP improvement following CPAP therapy. It is reasonable to believe that increased CPAP adherence may be the mediator. ${ }^{64}$

Adherence to CPAP is an important confounding variable which should be assessed, and newer CPAP devices have made it possible to measure hours of time at effective pressure, as well as whether residual apnea events persist. However, these devices do not measure any continuing, untreated burden of hypoxia and sympathetic stress, because 
they do not measure the time that the patient sleeps without wearing the device. Additionally, they do not measure sleep, so misleading data on residual disease burden may be obtained from patients who wear CPAP while awake. These measurement errors would be expected to bias the results toward the null, and attenuate any observed differences between treatment and control groups, so that a negative or small result does not imply the absence of benefit.

Some of the most significant decreases to date in daytime and nighttime blood pressure as a result of CPAP therapy come from a Spanish randomized controlled CPAP trial. Lozano et al studied 64 patients with resistant hypertension and an AHI $>15$. After 3 months, the group using CPAP in addition to their antihypertensive regimens demonstrated an approximately $5 \mathrm{mmHg}$ reduction in daytime BP compared with the group using only their medications. As in previous studies, this research demonstrated that increased CPAP adherence (CPAP use $>5.8$ hours/night) was associated with the most dramatic BP declines; 24 hour systolic BP fell nearly $10 \mathrm{mmHg}$ in the more adherent group. Notably, this trial also demonstrated a significant reduction in nocturnal nondipping patients in the CPAP group not reflected in the control group. Therefore, this recent study not only highlighted another group who might benefit most from CPAP therapy (patients with resistant hypertension) but also added to the growing knowledge base that treatment adherence matters in BP reduction and restoration of expected nocturnal dipping. ${ }^{65}$

Apart from severity of OSA and treatment adherence influencing CPAP's BP-lowering abilities, one might also consider the pathophysiology of hypertension when evaluating the earlier conflicting findings. As previously noted, OSA can lead to vasoconstriction through a variety of mechanisms including sympathetic over-activation and oxidative stress. When these processes persist for an extended period, vascular walls may develop at least partially irreversible fibrosis leading to elevated systemic BP over time. Trials examining the effects of CPAP on BP follow study participants for as little as 2 weeks and rarely more than 12 weeks. ${ }^{60}$ It is possible that this is simply not enough time to evaluate outcomes given the longstanding nature of OSA with resulting hypertension.

In general, the characteristics that predict the greatest likelihood of BP-lowering benefit from CPAP among patients with OSA are: (1) adherence to treatment, (2) baseline BP, with higher BP predicting greater response, (3) the presence of at least moderate to severe OSA, and (4) reversibility in disease-related vascular remodeling. However, even the most responsive patients may have some residual hypertension after prolonged CPAP therapy. While OSA-targeted therapy represents an attractive option for individuals with coexistent hypertension, antihypertensive medication usually continues to be necessary. Unfortunately, there is insufficient evidence to suggest which class of drugs is most effective in treating OSA-associated hypertension. There had been some promise from trials demonstrating partial restoration of the nocturnal BP dip with the use of beta-adrenergic blockers, though diuretics have been shown to be equally as efficacious. ${ }^{66}$ Given OSA's activation of the renin-angiotensin-aldosterone system, inhibitory medications such as angiotensin-converting enzyme (ACE) inhibitors and aldosterone antagonists are frequently used, albeit in the absence of direct evidence regarding their superior efficacy. In fact, the dry cough affecting some patients on ACE inhibitors may lead to airway inflammation and worsening of their OSA symptoms. ${ }^{67}$

\section{Implications for enhanced patient care and quality of life}

As noted previously, it has become increasingly well established that OSA is a major modifiable cause of hypertension; a continual challenge is to identify individuals at risk for the sleep disorder. Because $30 \%-40 \%$ of hypertensive patients have OSA, it behooves their healthcare providers to screen for it. The Berlin questionnaire is the most studied and best validated primary care-based assessment for the presence of OSA. ${ }^{68}$ Patients answer questions regarding known signs and symptoms of OSA such as obesity, snoring, excessive daytime sleepiness, witnessed breathing pauses while asleep, and the presence of hypertension. Based on their responses, individuals are stratified into "low risk" or "high risk" groups. More simplified questionnaires, such as the Graduated Apnea Screening Protocol (GASP), are also used in risk stratification but lack such rigorous validation.

Once patients are deemed at significant risk for OSA, overnight PSG is indicated to diagnose disease and quantify its severity. Traditionally completed in a sleep laboratory, home PSG is now becoming a viable alternative, facilitating diagnosis and treatment. Indeed, the Center for Medicare and Medicaid Services in America now covers the cost of CPAP therapy commencement based on home PSG data. While CPAP is the best studied treatment for OSA in terms of symptomatic improvement and BP control, other options include oral mandibular advancement devices or surgical interventions for those individuals with mild to moderate disease unable to tolerate CPAP.

Once appropriate therapy for OSA has been selected, the battle to minimize its sequelae begins. As we noted previously, CPAP adherence is a critical predictor of $\mathrm{BP}$ response. 
Patients benefit from regular follow-up at a comprehensive sleep center, which can not only optimize treatment adherence but can also address coexisting sleeping disorders contributing to further sleep disruption. This specialty care should proceed in conjunction with close primary care monitoring. Weight management strategies along with behavioral and lifestyle modifications, such as smoking cessation, are essential. Antihypertensive medications are often necessary adjuncts to CPAP therapy and are typically monitored in the primary care setting. With recent evidence showing that nighttime BP elevation correlates with cardiovascular risk, we might expect more antihypertensive administration to migrate toward evening. ${ }^{69}$

\section{Conclusion}

While ongoing research investigates OSA's association with a variety of cardiovascular disorders, its etiologic link with hypertension is the most convincing. There is now a large body of evidence showing that CPAP therapy lowers both nocturnal and daytime BP, especially in patients with moderate to severe OSA who adhere to treatment. The effects of treatment may be greatest if the patient has resistant hypertension, perhaps due to OSA's even stronger causal link to their disorder. Ongoing adherence with antihypertensive medication is a necessary, though not necessarily sufficient, strategy to lower BP into an acceptable range.

A multipronged approach to OSA-associated hypertension is needed due to its multifactorial nature. Inappropriately increased vascular tone and reactivity - due to sympathetic over activity and prolonged exposure to high levels of neurohumoral agents and neurotransmitters - perpetuates prolonged BP elevation well beyond periods of nocturnal hypoxemia. Alteration in the vascular wall structure itself is likely another end result of this imbalance, particularly if OSA is left unchecked for many years. Combating the incident development of hypertension in the OSA patient, then, argues for effective screening strategies to identify and treat latent cases of OSA as soon as possible, before vascular remodeling occurs and mitigates the potential BPlowering benefits of treatment of OSA. And among patients in whom OSA and BP are already established, a comprehensive regimen of CPAP therapy along with medical and behavioral management is most likely to garner benefits. This includes BP-lowering medication as well as a sustained program of long-term weight management. Given that 50\% of persons in America are expected to be obese by the year $2030,{ }^{70}$ prevention, early recognition, and prompt intervention of hypertension related to OSA should be the goals for all affected individuals, so that long term health and economic consequences may be mitigated.

\section{Disclosure}

The authors report no conflicts of interest in this work.

\section{References}

1. Chobanian AV, Bakris GL, Black HR, et al. The Seventh Report of the Joint National Committee on Prevention, Detection, Evaluation, and Treatment of High Blood Pressure: the JNC 7 report. JAMA. 2003;289:2560-2572.

2. Hajjar I, Kotchen Ta. Trends in prevalence, awareness, treatment, and control of hypertension in the United States, 1988-2000. JAMA. 2003; 290:199-206.

3. Ford ES, Giles WH, Dietz WH. Prevalence of the metabolic syndrome among US adults: Findings from the Third National Health and Nutrition Examination Survey. JAMA. 2002;287:356-359.

4. Young T, Palta M, Dempsey J, Skatrud J, Weber S, Badr S. The occurrence of sleep-disordered breathing among middle-aged adults. $N$ Engl J Med. 1993;328:1230-1235.

5. Nieto FJ, Young TB, Lind BK, et al. Association of sleep-disordered breathing, sleep apnea, and hypertension in a large community-based study. Sleep Heart Health Study. JAMA. 2000;283:1829-1836.

6. Dart RA, Gegoire JR, Gutterman DD, Woolf SH. The association of hypertension and secondary cardiovascular disease with sleepdisordered breathing. Chest. 2003;123:244-260.

7. Baguet JP, Narkiewicz K, Mallion JM. Update on hypertension management: obstructive sleep apnea and hypertension. J Hypertension. 2006;24:205-208

8. Iber C, Ancoli-Israel S, Chesson A, Quan SF, for the American Academy of Sleep Medicine. The AASM Manual for the Scoring of Sleep and Associated Events: Rules, Terminology and Technical Specifications. Westchester: American Academy of Sleep Medicine; 2007.

9. Gould G, Whyte K, Rhind G, et al. The sleep hypopnea syndrome. Am Rev Respir Dis. 1988;137:895-898.

10. Calhoun DA, Harding K. Sleep and hypertension. Chest. 2010;138: 434-443.

11. Kerkhof GA, Van Dongen HPA, Bobbert AC. Absence of endogenous circadian rhythmicity in blood pressure? Am J Hypertens. 1998; 11(3 Part 1):373-377.

12. Somers VK, Dyken ME, Mark AL. et al. Sympathetic-nerve activity during sleep in normal subjects. N Engl J Med. 1993;328:303-307.

13. Noda A, Okada T, Hayashi H, Yasuma F, Yokota M. 24-hour ambulatory blood pressure variability in obstructive sleep apnea syndrome. Chest. 1993;103:1343-1347.

14. Ancoli-Israel S, Strepnowsky C, Dimsdale J, Marler M, Cohen-Zion M, Johnson S. The effect of race and sleep-disordered breathing on nocturnal BP "dipping": analysis in an older population. Chest. 2002;122: $1148-1155$.

15. O'Brien E, Sheridan J, O'Malley K. Dippers and non-dippers. Lancet. 1988;332:397.

16. Ingelsson E, Bjorklund-Bodegard K, Lind L, et al. Diurnal blood pressure pattern and risk of congestive heart failure. JAMA. 2006;295: 2859-2866.

17. Timio M, Venanzi S, Lolli S, et al. "Non-dipper" hypertensive patients and progressive renal insufficiency: a 3-year longitudinal study. ClinNephrol. 1995;43:382-387.

18. Dolan E, Stanton AV, Thorn S, et al; ASCOT Investigators. Ambulatory blood pressure monitoring predictions cardiovascular events in treated hypertensive patients - an Anglo-Scandinavian cardiac outcomes trial substudy. J Hypertens. 2009;27:876-885.

19. Ohkubo T, Hozawa A, Nagai K, et al. Prediction of stroke by ambulatory blood pressure monitoring versus screening blood pressure measurements in a general population: the Ohasama study. $J$ Hypertens. 2000;18:847-854. 
20. Law MR, Morris JK, Wald NJ. Use of blood pressure lowering drugs in the prevention of cardiovascular disease: metaanalysis of 147 randomised trials in the context of expectations from prospective epidemiological studies. BMJ. 2009;228:1245-1253.

21. Cook NR, Cohen J. Herbert PR, Taylor JO, Hennekens CH. Implications of small reductions in diastolic blood pressure for primary prevention. Arch Intern Med. 1995;155:701-709.

22. Gangwisch JE, Heymsfield SB, Boden-Albala B, et al. Short sleep duration as a risk factor for hypertension: analyses of the first National Health and Nutrition Examination Survey. Hypertension. 2006;47: 833-839.

23. Knutson KL, Van Cauter E, Rathouz PJ, et al. Association between sleep and blood pressure in midlife: the CARDIA sleep study. Arch Intern Med. 2009;169:1055-1061.

24. Calhoun DA, Harding SM. Sleep and hypertension. Chest. 2010;138: 434-443.

25. Brooks D, Horner RL, Kozar F, Render-Teixeira CL, Phillipson EA. Obstructive sleep apnea as a cause of systemic hypertension. Evidence from a canine model. J Clin Invest. 1997;99:106-109.

26. Leuenberger UA, Brubaker D, Quaraishi S, et al. Effects of intermittent hypoxia on sympathetic activity and blood pressure in humans. Auton Neurosci. 2005;121:87-93.

27. Young T, Shahar, E, Nieto FJ, et al. Predictors of sleep-disordered breathing in community-swelling adults: the Sleep Heart Health Study. Arch Intern Med. 2002;162:893-900.

28. Endeshaw YW, White WB, Kutner, M, Ouslander JG, Bliwise DL. Sleep-disordered breathing and 24-hour blood pressure pattern among older adults. $J$ Gerontol. 2008;64A:280-285.

29. Fletcher ED, Debehnke RD, Lovoi MS, et al. Undiagnosed sleep apnea in patients with essential hypertension. Ann Intern Med. 1985;103: 190-195.

30. Lavie P. Ben-Yosef-R. Rubin AE. Prevalence of sleep apnea syndrome among patients with essential hypertension. Am Heart J. 1984;108 373-376.

31. Worsnop CJ, Naughton MT, Barter CE, Morgan TO, Anderson AI, Pierce RJ. The prevalence of obstructive sleep apnea in hypertensives. Am J Respir Crit Care Med. 1998;157:111-115.

32. Pimenta E, Calhoun DA. Primary aldosteronism: diagnosis and treatment. J Clin Hypertens. 2006;8:887-893.

33. Dudenbostel T, Calhoun DA. Resistant hypertension, obstructive sleep apnoea and aldosterone. J Hum Hypertens. June 9, 2011. [Epub ahead of print.]

34. Kapur VK, Resnick HE, Gottlieb DJ. Sleep disordered breathing and hypertension: does self-reported sleepiness modify the association? Sleep. 2008;31:1127-1132.

35. Bixler EO, Vgontzas AN, Lin HM, et al. Association of hypertension and sleep-disordered breathing. Arch Intern Med. 2000;160:2289-2295.

36. Horne RS, Yang JS, Walter LM, et al. Elevated blood pressure during sleep and wake in children with sleep-disordered breathing. Pediatrics. 2011;128:e85-e92.

37. Lavie P, Herer P, Hoffstein V. Obstructive sleep apnea syndrome as a risk factor for hypertension: population study. $B M J$. 2000;320: 479-482.

38. Young T, Peppard P, Palta M, et al. Population-based study of sleepdisordered breathing as a risk factor for hypertension. Arch Intern Med. 1997;157:1746-1752.

39. Peppard P, Young T, Palta M, Skatrud J. Prospective study of the association between sleep-disordered breathing and hypertension. NEJM. 2000;342:1378-1384.

40. O'Connor GT, Caffo B, Newman AB, et al. Prospective study of the association between sleep-disordered breathing and hypertension: the Sleep Heart Health Study. Am J RespirCrit Care Med. 2009;179: 1159-1164.

41. Cano-Pumarega I, Duran-Cantolla J, Aizpuru F, et al. Obstructive sleep apnea and systemic hypertension: longitudinal study in the general population. Am J RepirCrit Care Med. August 25, 2011. [Epub ahead of print.]
42. Peppard PE. Is obstructive sleep apnea a risk factor for hypertension? Differences between the Wisconsin Sleep Cohort and the Sleep Heart Health Study. Journ Clin Sleep Med. 2009;5:404-405.

43. Philips BG, Narkiewicz K, Pesek CA, et al. Effects of obstructive sleep apnea on endothelin-12 and blood pressure. J Hypertens. 1999;17: 61-66.

44. Carlson J, Rangemark C, Hedner J. Attenuated endothelium-dependent vascular relaxation in patient with sleep apnoea. J Hypertens. 1996;14: $577-584$.

45. Alzoghaibi MA, Bahammam AS. The effect of one night of continuous positive airway pressure therapy on oxidative stress and antioxidant defense in hypertensive patients with severe obstructive sleep apnea. Sleep Breath. May 13, 2011. [Epub ahead of print.]

46. Ip MSM, Lam B, Chan L-Y, et al. Circulating nitric oxide is suppressed in obstructive sleep apnea and is reversed by nasal continuous positive airway pressure. Am J Resp Crit Care Med. 2002;165:1624-1628.

47. Schultz R, Schmidt D, Blum A, et al. Decreased plasma levels of nitric oxide derivatives in obstructive sleep apnoea: response to CPAP therapy. Thorax. 2000;55:1046-1051.

48. Bayram NA, Ciftci B, Keles T, et al. Endothelial function in normotensive men with obstructive sleep apnea before and 6 months after CPAP treatment. Sleep. 2009;32:1257-1263.

49. Weiss JW, Liu MD, Huang J. Physiological basis for a causal relationship of obstructive sleep apnoea to hypertension. Exp Physiol. 2007;92:21-26.

50. Kraiczi H, Hedner J, PekerY, Carlson, J. Increased vasoconstrictor sensitivity in obstructive sleep apnea. J Appl Physiol. 2000;89:493-498.

51. Mills PJ, Kennedy BP, Loredo JS, et al. Effects of nasal continuous positive airway pressure and oxygen supplementation on norepinephrine kinetics and cardiovascular response in obstructive sleep apnea. $J$ Appl Physiol. 2006;100:343-348.

52. Philips CL, Yang Q, Williams A. et al. The effect of short-term withdrawal from continuous positive airway pressure therapy on sympathetic activity and markers of vascular inflammation in subjects with obstructive sleep apnoea. J Sleep Res. 2007;16:217-225.

53. Narkiewicz K, Kato M, Phillips BG, et al. Nocturnal continuous positive airway pressure decreased daytime sympathetic traffic in obstructive sleep apnea. Circulation. 1999;100:2332-2335.

54. Norman D, Loredo JS, Nelesen RA, et al. Effects of continuous positive airway pressure versus supplemental oxygen on 24-hour ambulatory blood pressure. Hypertension. 2006;47:840-845.

55. Sullivan C, Issa F, Berthon-Jones M, and Eves L. Reversal of obstructive sleep apnea by continuous positive airways pressure applied through the nares. Lancet. 1982;1:862-865.

56. Bazzano LA, Khan Z, Reynolds K, He J. Effect of nocturnal nasal continues airway pressure on blood pressure in obstructive sleep apnea. Hypertension. 2007;50:417-423.

57. Zhao Q, Zhi-hong L, Luo Q, et al. Effects of continuous positive airway pressure on blood pressure and daytime sleepiness in obstructive sleep apnea patients with coronary artery disease under optimal medications. Sleep Breath. February 22, 2011. [Epub ahead of print.]

58. Alajmi M, Mulgrew AT, Fox J, et al. Impact of continuous airway pressure therapy on blood pressure in patients with obstructive sleep apnea hypopnea: a meta-analysis of randomized controlled trials. Lung. 2007; 195:67-72.

59. Mo L, He QY. Effect of long-term continuous positive airway pressure ventilation on blood pressure in patients with obstructive sleep apnea hypopnea syndrome: a meta-analysis of clinical trials. Zhonghua YiXue ZaZhi. 2007;87:1177-1180.

60. Haentjens P, Van Meerhaeghe A, Moscariello A, et al. The impact of continuous airway pressure on blood pressure in patients with obstructive sleep apnea syndrome: evidence from a meta-analysis of placebocontrolled randomized trials. Arch Intern Med. 2007;167:757-764.

61. Durán-Cantolla J, Aizpuru F, Montserrat J, et al; SpanishSleep and BreathingGroup. Continuous positive airway pressure as treatment for systemic hypertension in people with obstructive sleep apnoea: randomized controlled trial. BMJ. 2010;341:c5991. 
62. Turnbull; Blood Pressure Lowering Treatment Trialists' Collaboration. Effects of different blood-pressure-lowering regimens on major cardiovascular events: results of prospectively designed overviews of randomized trials. Lancet. 2003;362(9395):1527-1535.

63. Weaver TE, Grunstein RR. Adherence to continuous positive airway pressure therapy: the challenge to effective treatment. Proc Am Thorac Soc. 2008;5:173-178.

64. Robinson GV, Langford BA, Smith DM, Stradling JR. Predictors of blood pressure fall with continuous positive pressure (CPAP) treatment of obstructive sleep apnea (OSA). Thorax. 2008;63:855-859.

65. Lozano L, Tovar JL, Sampol Gabriel, et al. Continuous positive airway pressure treatment in sleep apnea patients with resistant hypertension: a randomized, controlled trial. J Hypertension. 2010;28:2161-2168.

66. Kraiczi H, Hedner J, Peker Y, Grote L. Comparison of atenolol, amlodipine, enalapril, hydrocholorothiazide, and losartan for antihypertensive treatment in patients with obstructive sleep apnea. Am J Respir Crit Care Med. 2000;161:1423-1428.
67. Cicolin A, Mangiardi L, Mutani R, Bucca C. Antiotensin-converting enzyme inhibitors and obstructive sleep apnea. Mayo Clin Proc. 2006;81:53-55.

68. Netzer NC, Stoohs RA, Netzer CM, Clark K, Strohl KP. Using the Berlin Questionnaire to identify patients at risk for the sleep apnea syndrome. Ann Intern Med. 1999;131:485-491.

69. Hermida RC, Ayala DE, Mojon A, Fernandez JR. Decreased sleeptime blood pressure determined by ambulatory monitoring reduces cardiovascular risk. Hypertension. 2011;58:1165-1173.

70. Wang YC, McPherson K, Marsh T, Gortmaker SL, Brown M. Health and economic burden of the projected obesity trends in the USA and the UK. Lancet. 2011;378:815-825.

\section{Publish your work in this journal}

ChronoPhysiology and Therapy is an international, peer-reviewed, open access journal focusing on research into the cyclic variations and rhythmicity in physiological processes in the body and the research and development and optimal timing of administration of therapeutic targets to achieve improved outcomes and quality of life for the patient. The

\section{Dovepress}

manuscript management system is completely online and includes a very quick and fair peer-review system. Visit http://www.dovepress.com/ testimonials.php to read real quotes from published authors. 